\title{
Marburg virus disease
}

\author{
G. A. MARTini \\ M.D. \\ University of Marburg, West Germany
}

\section{Summary}

In the late summer of 1967 an epidemic in thirty-one patients in Germany and Yugoslavia of a disease transmitted from African green monkeys occurred; seven patients died. The incubation period was from 4 to 7 days. The main clinical features were headache, high fever, diarrhoea, a very characteristic rash, severe bleeding tendency and involvement of the central nervous system. Nearly all organs were involved and showed severe cell necroses. The aetiological agent was identified as an RNS-virus and was named Marburg virus. It was detected in the blood, urine, throat-washing and seminal fluid.

IN August 1967 a hitherto unknown severe infectious disease with features of haemorrhagic fever was observed simultaneously in Germany and Yugoslavia which could be traced to imported monkeys (Cercopithecus aethiops) from Uganda.

Until then only single cases of diseases transmitted by monkeys were known (e.g. herpes simiae, rabies, hepatitis). The new disease was the first to occur as an epidemic.

\section{Incidence}

Altogether thirty-one persons (twenty males, eleven females) contracted the disease: at Marburg twenty-three, at Frankfurt six, and at Belgrade two persons. Out of these thirty-one patients seven died.

The epidemic appeared in August and ended in November 1967. Before and afterwards no more cases were seen.

\section{Clinical picture (Figs. 1 and 2)}

The incubation period could be determined in some patients who had only once been exposed to the infectious material; it varied from 3 to 9 days.

The prodromal phase was very short. The disease began rather abruptly with extreme malaise, pains in the limbs, and severe frontal and temporal headache.
Within a few hours the fever increased to $39^{\circ} \mathrm{C} 3$ without rigors and reached a maximum on the $3 \mathrm{rd}+0$ and 4th days; the temperature fell gradually after- or wards with a second peak between the 12th and 20th $\perp$ days.

Relative bradycardia occurred, especially in the $\stackrel{A}{N}$ early days, whereas tachycardia was only found in 은 the fatal cases. Many patients complained from the beginning about nausea and suffered from frequent, and occasionally uncontrollable, vomiting. Waterye

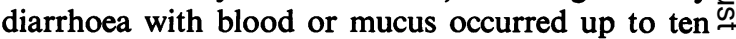
times daily with symptoms and signs of severe $\vec{\varphi}$ dehydration and acute renal failure.

All patients developed a characteristic maculo papular rash on the 5th to 8th days. It began o. the face and on the buttocks, then progressed to the trunk and extremities. Most characteristic were dark red pinhead papules round the hair follicles. After 1 or 2 days it developed into a sharply de- $\stackrel{\varrho}{\Rightarrow}$ lineated maculo-papular lesion which coalesced into a more diffuse and dark red erythema. Cutaneous purpura was rare. The rash was in many patients accompanied by scrotal dermatitis or erythema of the greater labia. About the end of the second week all patients peeled, especially on the palms and soles.

The external rash was in most cases accompanied 3 by an enanthem with dark red colouring of the soft palate and tapioca-like transparent lesions.

A conjunctivitis and photophobia developed in half of the patients. Swelling of the lymph nodes was observed in the nuchal, cervical, and axillary regions between the 3rd and 6th days of illness. The lymph nodes were soft and sensitive to pressure. The $N$ spleen was palpable in only one patient, the liver $N$ was tender but not enlarged. The central nervous N system was affected in different ways. Nearly all $\bar{\sigma}$ patients showed a sullen, slightly aggressive, and negativistic behaviour. Those who died became restless, confused, unconscious, and died in deep coma. Two had severe convulsions. Some patients complained of paraesthesia and restless legs. One $\overline{0}$ patient developed a severe post-infectious myelitis 


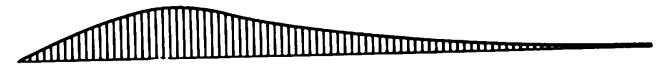

Vomiting

$(n=21)$

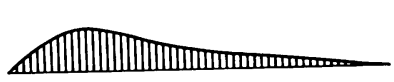

Diarrhoea

$(n=19)$

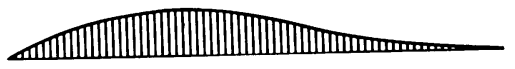

Lymphadenopathy

$(n=10)$

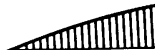
Enanthem
$(n=17)$
Rash
$(n=23)$
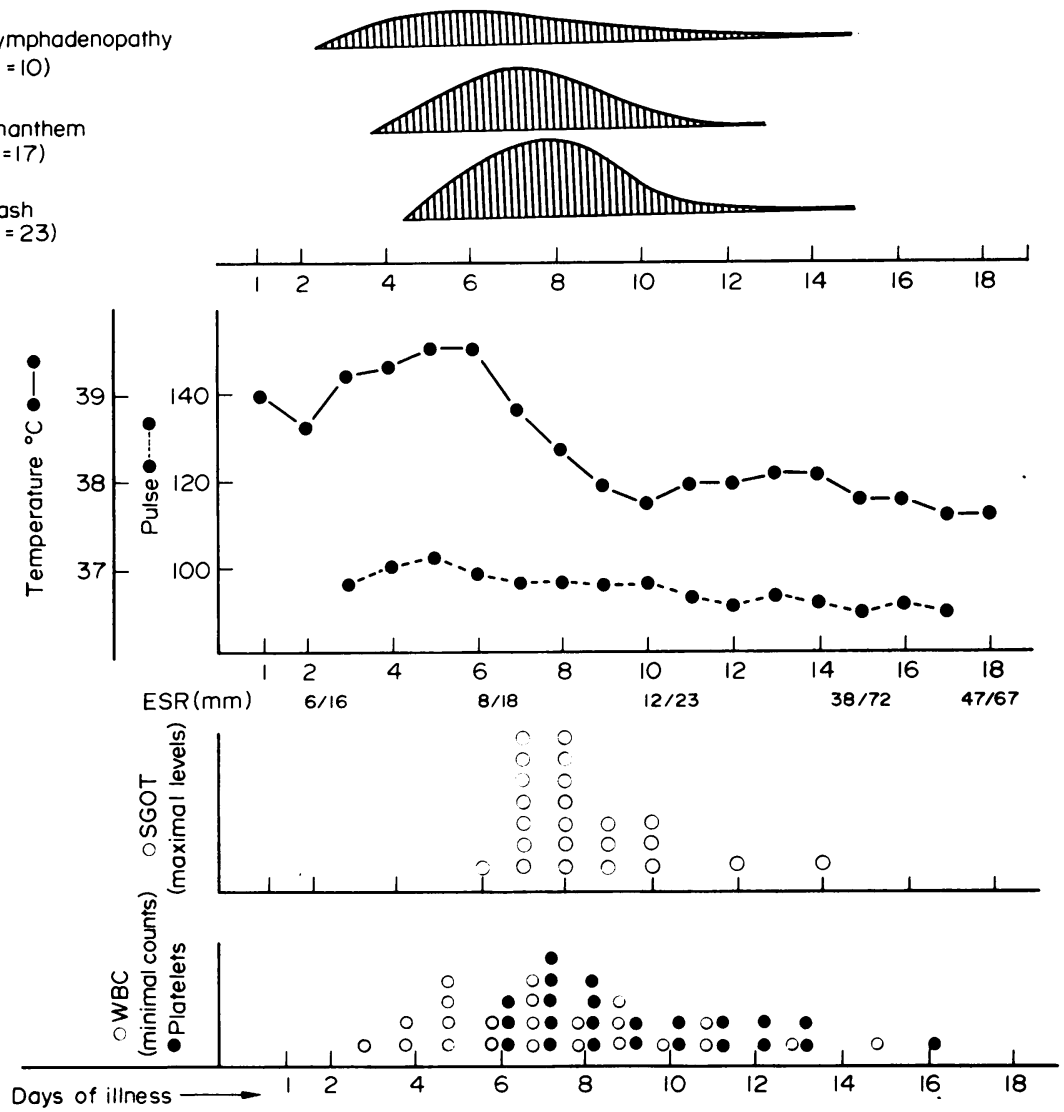

FIG. 1. Incidence and duration of main signs and symptoms. Height of curves in top part is determined by the number of patients with listed symptom on a given day. For SGOT, WBC and platelet counts, the figure indicates how many patients had reached maximal or minimal levels on the stated day. (Courtesy of the Editor, Deutsche medizinische Wochenschrift.)

with a Landry-type of paralysis with albuminocellular dissociation in the cerebrospinal fluid (Guillain-Barré syndrome). One patient became psychotic several weeks after the acute illness.

About half of the patients exhibited marked haemorrhagic diathesis with spontaneous bleeding from the nose, gingiva and gastro-intestinal tract, and haematuria. The women had genital bleeding. At the sites of injections and needle punctures severe bleeding occurred.

The pancreas was affected in several patients as shown by the increased serum amylase values. 


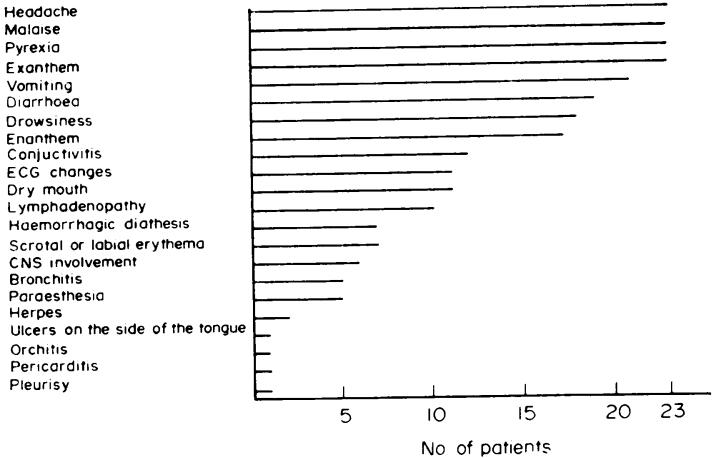

FIG. 2. Incidence of symptoms and signs. (Courtesy of the Editor, Deutsche medizinische Wochenschrift.)

Nearly all patients had signs of severe hepatocellular damage with an extreme increase of the transaminase levels. Neither jaundice nor true hepatic failure developed. In the electrocardiogram alterations compatible with diffuse myocarditis and disturbances of cardiac rhythm were seen. In many patients cardiac failure developed. Nearly all of the patients who died had severe kidney involvement with acute renal failure. Those who survived had longer periods of polyuria. The haemopoetic system was severely damaged in nearly all patients as could be recognized by marked leukopenia and severe thrombocytopenia.

\section{Course of the disease}

The illness lasted 15-20 days. In some cases there was a relapse. Five patients showed a further increase of serum transaminase at this time with an increased temperature. The convalescent period was very drawn out. There were disturbances of the autonomic nervous system with exhaustion and increased perspiration. Some of the patients had a considerable loss of hair and complained of tenderness of the liver and alcohol intolerance. Unilateral testicular atrophy was observed in five patients. Some had reduced libido and potency and oligospermia; the ketosteroid levels were normal. Three women were delivered of four healthy babies with no signs of disease.

\section{Laboratory findings}

The erythrocyte sedimentation rate rarely exceeded the upper normal range. The haematologic changes apart from leukopenia and thrombocytopenia were characterised by a shift to the left in the granulocytes with up to $40 \%$ stab cells, promyelo-, metamyelo- and myelocytes. This shift to the left was accompanied by the appearance of so-called degenerative granulocytes and Pelger-like cells. There was a marked increase of atypical lymphocytes, plasma cells, immunoblasts and so-called pyronino-气 phil blast cells, up to $15 \%$ of the total count. Bone marrow puncture showed an elevated number of immature megakariocytes. All patients developed $\leftarrow$ severe thrombocytopenia with sometimes less than $10,000 / \mathrm{mm}^{3}$. At the same time the thrombin time was prolonged, indicating consumptive coagulopathy $\frac{}{0}$ In none of ten patients in whom the prothrombines time, partial thromboplastin time and fibrinogen were measured did the alterations and plasma coagulation factors explain the severe haemorrhagics diathesis.

The extreme increase of serum transaminases andother liver enzymes reflected the severe hepato-. cellular damage. In four of the five patients who diedo the transaminases increased up to $3000-6000 \mathrm{U} / \mathrm{l}$ The relation between SGOT and SGPT was $7: 1$. The maximum increase was between the 7 th and 8 th days, with a second peak around the 35th day in three patients. The bilirubin was only slightlyor increased in some patients with anuria. The creatinin and urea were elevated in those patients who dieco with renal failure. The total serum protein decreased markedly in several patients.

The other complications included bronchoes pneumonia (five cases), leg oedema (five cases) orchitis (three cases), post-infectious myelitis (onecase), and psychosis (one case).

\section{Pathology}

In nearly all organs areas of focal necrosis without much inflammatory reaction were seen. They wereo particularly obvious in the liver, in the lymphatico system, in the spleen, in the testicles and ovaries, and in the pancreas. In addition the lymphatic tissue showed plasmacellular, monocytoidal trans? formation. Together with the areas of necrosis socalled basophilic bodies were noticed near the necrotico cells or as inclusion bodies in parenchymal cells $\stackrel{3}{?}$. Those patients who died showed glial nodule encephalitis in all areas of the brain and serious parenchymal damage of the kidneys with definite signs of tubular insufficiency and haemorrhagic diathesis in all organs.

\section{Diagnosis}

Laboratory methods included the direct isolation of the agent in the blood, organ tissues, throap washings, urine and semen (Fig. 3). Virus antigenw was made visible by means of immuno-fluorescence? Direct electron microscopic demonstration of the virus was possible after centrifugation of serum on tod carrier films.

The morphology of the Marburg virus is most unusual. It differs in size and shape from most known 


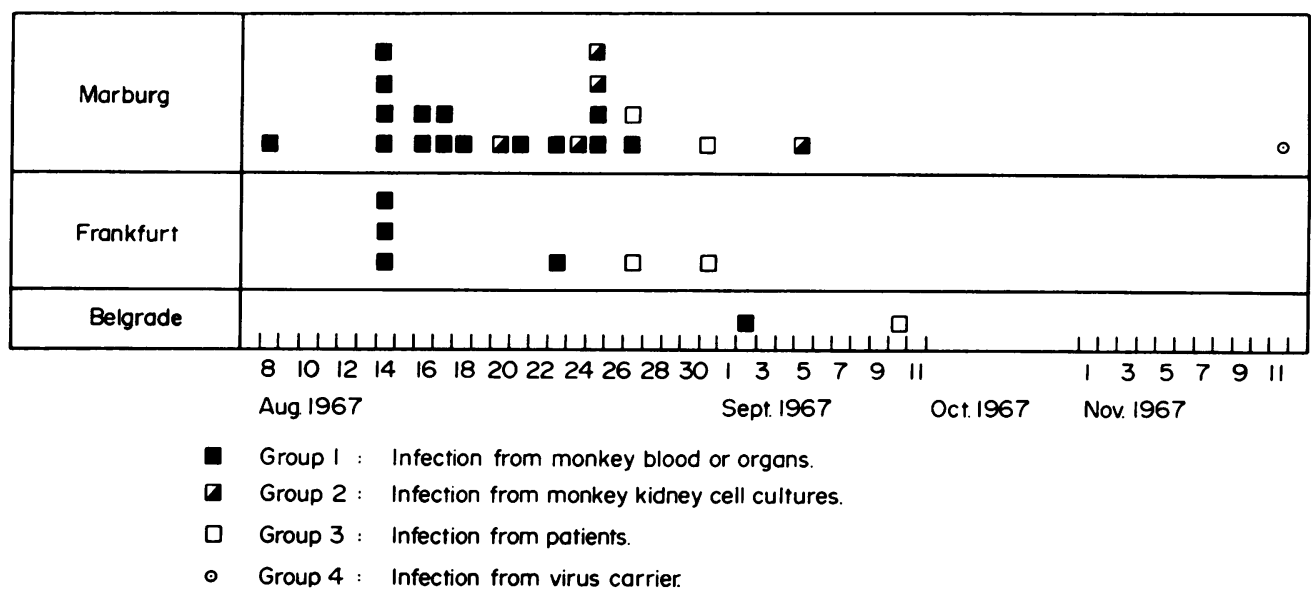

Fig. 3. Onset of Marburg virus disease in thirty-one patients. (Courtesy of Professor Siegert, Marburg, and Springer Verlag, Heidelberg.)

viruses. The mean length is about $665 \mathrm{~nm}$. It shares certain similarities with other viruses (plant viruses, Rabies, Egtred virus of rainbow trout).

A complement fixing antibody test using a cell culture antigen from infected Vero carrier cells is highly specific.

\section{Epidemiology}

There were four groups of infection (Fig. 4). The first and largest group consisted of persons who had direct contact with blood and organs of monkeys imported from Uganda. The second group consisted of laboratory workers who had contact with simian organs or cell culture material. The third group contracted the infection from other patients' blood.

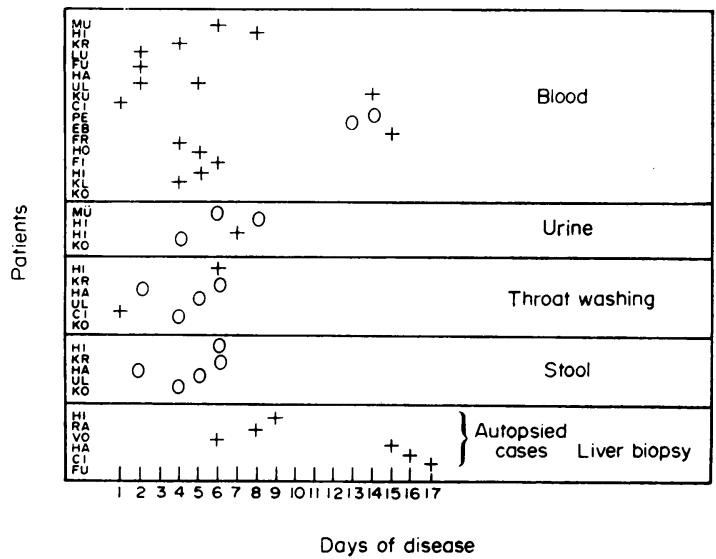

FIG. 4. Isolation of the virus from blood, urine, throat washing and liver biopsy material. (Courtesy of Professor Siegert, Marburg.)
Exposure in the household and family surroundings remained without consequences. In one woman a venereal way of transmission was the most probable, since her husband was found to excrete the Marburg virus in his semen.

The simultaneous outbreak of the disease in Marburg, Frankfurt and Belgrade was traced to common imported monkeys of the species Cercopithecus aethiops from Uganda. These monkeys were flown because of the Near East crisis, to Germany and Yugoslavia via London, where they were kept at the airport together with other animals. Seroepidemiologic studies using a complement-fixing antibody test seemed to suggest a high percentage of positive reactions in monkeys from Uganda and from other areas. These results could not be confirmed by other authors using a more specific method. The reservoir and method of distribution of the Marburg virus are still unknown. Intrathoracic multiplication of the virus was possible in Aedes aegypti.

\section{Treatment}

The therapy was symptomatic. Antibiotics were used prophylactically against secondary bacterial infections. Acute renal failure was treated according to standard rules including peritoneal dialysis. Low sodium human albumin was infused in patients with hypoproteinaemia, and electrolytes and fluid supplemented. The most difficult problem was the treatment of the haemorrhagic diathesis. Fresh blood thrombocyte concentrates, fibrinogen, $\varepsilon$-aminocaproic acid, vitamin $\mathrm{K}$ and the French preparation PPSB (Prothrombin, Proconvertin, Stuart-factor and antihaemophilicglobin B) were given. The latter proved to be the most efficient. 


\section{Course and prognosis}

The disease varied greatly in severity. There were some mild cases but most of the patients were critically ill. The fatality rate was $22 \%$. Complete recovery was the rule, but most patients even now after 5 years complain about easy fatiguability, inability to concentrate, loss of libido, etc. It is very difficult to decide what complaints have a real background or which symptoms are due to the unique situation, as all infections were acknowledged as occupational hazards and the pension scheme makes it necessary that follow-up studies are required every year. So a kind of post-Marburg virus disease neurosis might play a role.

\section{References}

GoRdon-Smith, C.E. (1971) Lessons from Marburg disease. In: The Scientific Basis of Medicine. Annual Review (Ed. by Ian Gilliland and J. Francis). Athlone Press, London.
Martini, G.A., KNaUfF, H., Schmidt, H., MaYer, G. \&ِ BALTZER, G. (1968) A hitherto unknown infectious disease contracted from monkeys. German Medical Monthly, $13 \stackrel{\mathbb{Q}}{\Omega}$ 457. Deutsche medizinische Wochinschrift, 93, 559.

MARTINI, G.A. \& SCHMIDT, H.A. (1968) Spermatogene Übertragung des Marburg Virus. Klinische Wochenschrift? 46, 391 .

MARTINI, G.A. \& SIEGERT, R. (Eds.) (1972) Marburg Virus Disease. Springer Verlag, Berlin, Heidelberg, New York

SIEGERT, R. (1972) Marburg Virus. Virology Monographs vol. 11, pp. 97-154. Springer Verlag, Berlin.

Stille, W., Böhle, E., Helm, E., Van Rey, W. \& Siede, Wל (1968) An infectious disease transmitted by Cercopithecus aethiops (Green Monkey disease). German Medical Monthly, 13, 470. Deutsche medizinische Wochenschrift, 0 93, 572 . 